Pensamiento Crítico Vol. 18 № 1, pp. 159-168

\title{
Metas de Inflación y Efectividad de la Politica Monetaria en el Perú
}

\author{
Richard Roca Garay ${ }^{1}$
}

\section{RESUMEN}

El presente artículo revisa el desempeño del control de la política monetaria para controlar la inflación en el Perú en comparación a las del resto del mundo y encuentra que la inflación en el Perú estuvo a un nivel medio. Ni muy alto, ni muy bajo, comparado a nivel de inflación de otros países que también aplican el esquema de metas de inflación.

Palabras Claves: Inflación, política monetaria, metas de inflación

\begin{abstract}
This paper reviews the performance of monetary policy to control inflation in Peru compared to the rest of the world and finds that inflation in Peru was at a medium level. Not too high, not too low compared to the inflation level of other countries that apply the inflation targeting framework.
\end{abstract}

Keywords: Inflation, monetary policy, inflation targeting

1 Profesor Principal de la Facultad de Economía UNMSM. Magister en Economía por la Pontificia Universidad Católica del Perú, y, Candidato a Doctor. Correo: rhroca@yahoo.com 


\section{Pensamiento Crítico Vol. 18. $\mathrm{N}^{\circ}$ I}

\section{INTRODUCCIÓN}

La Política Monetaria es el manejo de la oferta monetaria o de la tasa de interés por parte de la autoridad monetaria normalmente denominado como Banco Central. Los cuales tienen como objetivo central la Estabilidad de Precios, o sea, un bajo nivel de inflación. En nuestro país el Banco Central de Reserva del Perú (BCRP) es una entidad independiente y autónoma del Gobierno Central.

Existen diversos tipos de política monetaria. En el caso peruano, el objetivo fundamental señalado por la Constitución es la estabilidad monetaria, o sea que los precios sean estables.

Durante la década de los 90 el BCRP aplicaba políticas de controles monetarios tratando de administrar la tasa de crecimiento de la oferta monetaria para reducir la tasa de inflación llevando la tasa de inflación de más de 7,650\% en 1990 a 3.7\% en el 2000 como se puede apreciar en la siguiente figura.

Figura 1. Evolución de la Inflación. Perú 1980-2012

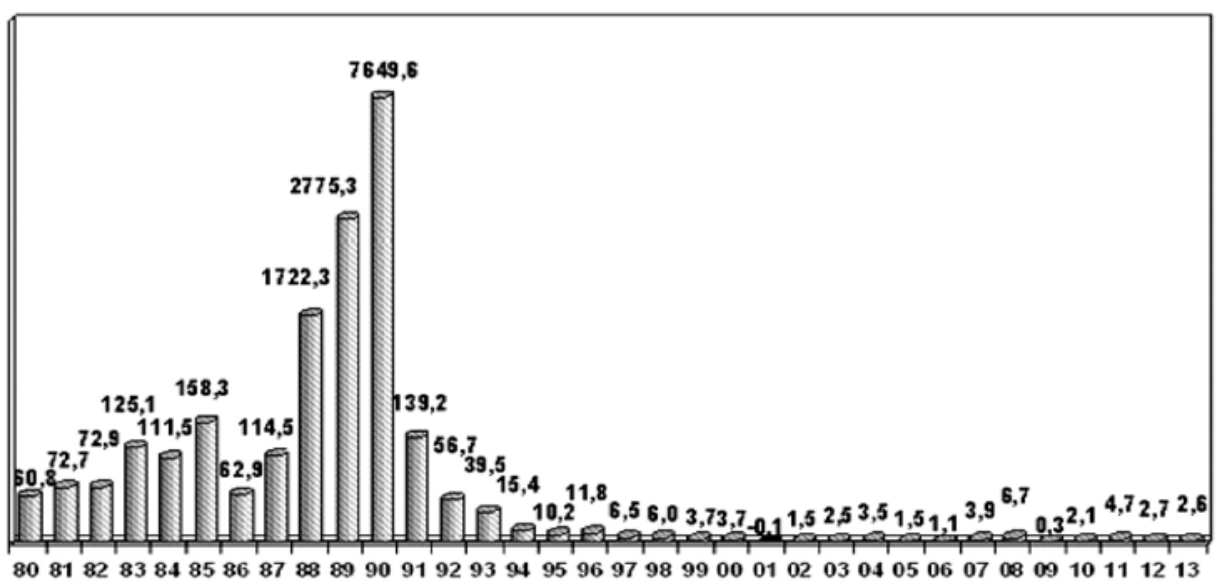

Fuente: INEI. 


\section{Richard Roca Garay}

\section{Metas de Inflación}

Desde el 2002 el BCRP, aplica el esquema Metas Explícitas de Inflación la cual consiste en anunciar una meta de inflación que el BCRP se compromete a alcanzar. Inicialmente dicha meta estaba entre $1.5 \%$ y $3.5 \%$ anual. El 2006 se modificó y desde el nuevo rango meta se ubica entre $1 \%$ y $3 \%$.

Una de las estrategias principales es calmar las expectativas del público y anclarla a la meta de inflación. Se aplicó por primera vez en Nueva Zelanda en 1990 y su uso se extendió a más de 20 países entre los que se encuentran Australia, Brasil, Canadá, Chile, Colombia, Corea, Israel, México, Polonia, Reino Unido, República Checa, Suecia y Turquía entre otros. Algunos países fijan una meta de inflación puntual, otros usan un rango meta como Perú pero en rangos diferentes y cada país con rangos meta distintos.

Bajo el esquema de Metas de Inflación la oferta monetaria es endógena siendo el instrumento más importante de política monetaria la tasa de interés de referencia (TR), definida como la tasa de interés que el Banco Central fija cada mes y que es el promedio de las tasas de interés que el BCR cobra los bancos comerciales (Tasa de descuento o Tasa de Regulación) y la tasa de interés pasiva o Tasa Overnight que paga el BCR a los Bancos Comerciales por sus depósitos en el BCR.

Figura 2. Tasas de Referencia, de Regulación y Overnight

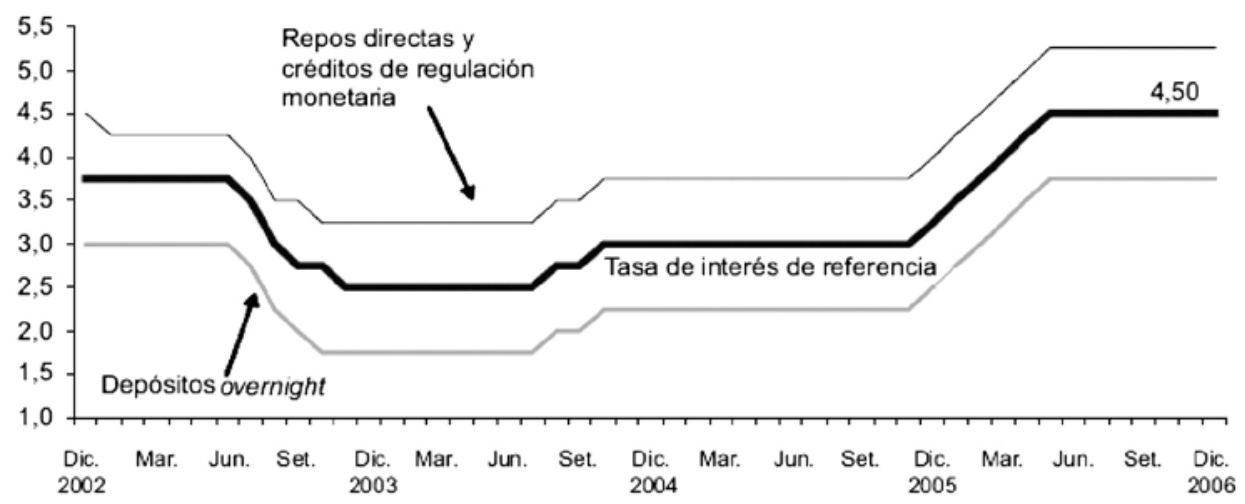

Fuente: Memorias del BCRP 2006. 


\section{Pensamiento Crítico Vol. 18. $\mathrm{N}^{\circ}$ I}

La Tasa de Referencia influye decisivamente en la tasa de interbancaria la cual a su vez influye en las diversas tasas que los bancos cobran a las empresas y familias a las cuales presta. A su vez estas tasas afectan a las decisiones de consumo, de inversión, también a las decisiones de entrada de capitales lo que influye a su vez en el tipo de cambio afectando a las exportaciones netas. Por tanto la tasa de referencia influye en la Demanda Agregada y mediante ello al nivel de precios. La figura 3 muestra los mecanismos de transmisión de la Metas Explicitas de Inflación en el Perú:

Figura 3. Esquema la influencia de la Política Monetaria sobre la Inflación

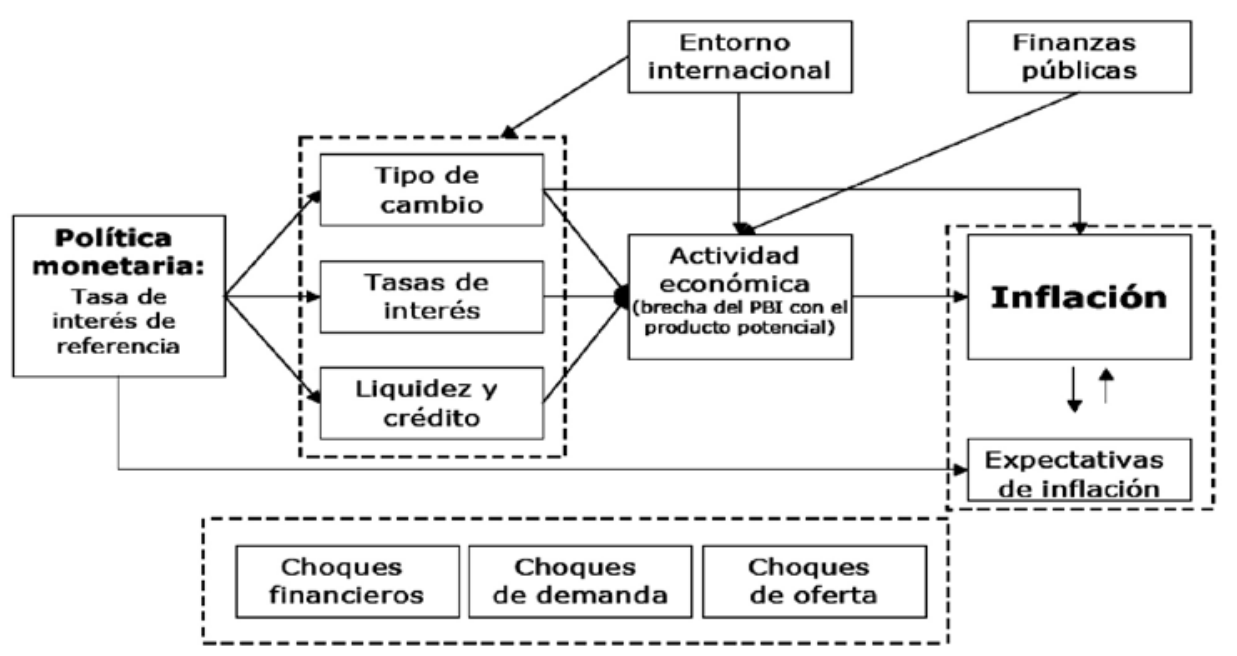

Fuente: BCRP

Si el BCRP cree que hay presiones inflacionarias que llevarían a que la inflación desborde el rango meta el BCRP incrementa la TR ello tiende a subir las Tasas de interés que los Bancos comerciales cobran a sus clientes con lo cual baja el consumo, baja la inversión, además induce a que los agentes quieran pasar de dólares a soles. La menor demanda de dólares hace bajar el valor de dicha moneda generando un impacto negativo en las exportaciones netas por lo tanto también en un crecimiento más lento de la Demanda Agregada. Así, tendríamos un mecanismo para que el BCRP influya sobre los precios para que la inflación se ubique en el rango meta. 


\section{Richard Roca Garay}

Adicionalmente el BCRP también tiene otros instrumentos como la tasa de encaje, la cual se usa solo en casos especiales, en situaciones que podría considerarse como de emergencia. La TR es la más relevante para el control de la inflación.

La Figura 4 muestra la evolución de la tasa de inflación desde enero del 2002 hasta noviembre el 2012 frente a sus rangos representadas por las líneas horizontales segmentadas y se puede observar que en muchos meses la inflación estuvo fuera de los niveles metas correspondientes lo que implica que la política monetaria no fue muy efectiva en mantener la inflación dentro de los rangos establecidos.

Figura 4. Inflación, Inflación Subyacente y No Subyacente

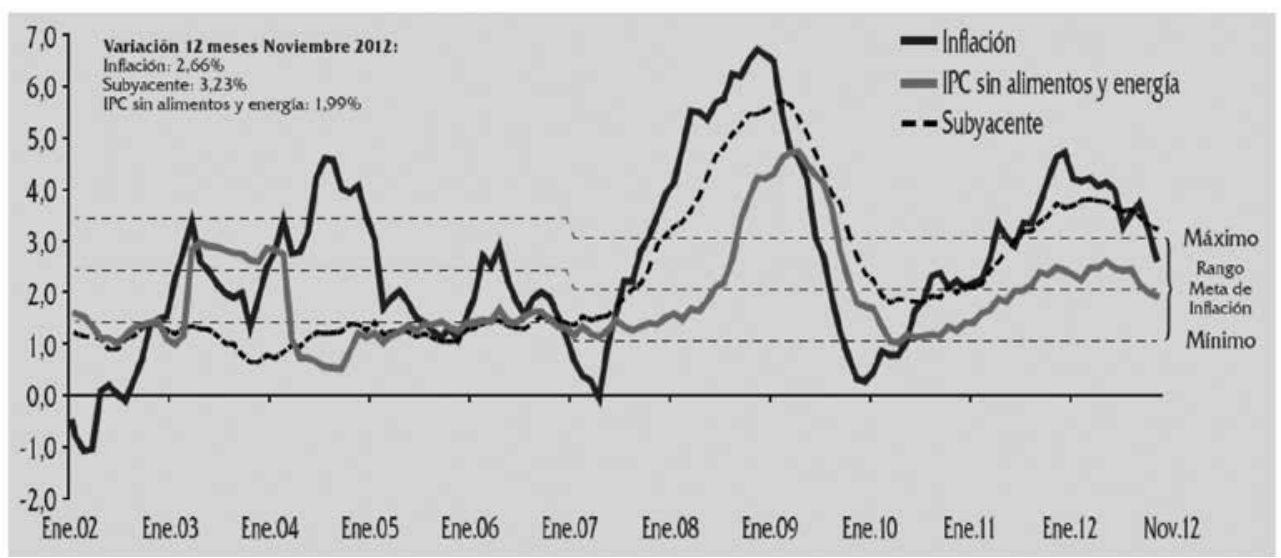

Fuente: BCRP Informe de la Inflación. Diciembre 2012

\section{Metas Explicitas de Inflación en el Mundo}

A nivel internacional son muchos los países que han adoptado el esquema de metas de inflación como lo muestra la siguiente tabla. Por oto lado, todos los países no tienen el mismo nivel de inflación objetivo y algunos mas bien tiene un rango meta (en vez de un nivel meta puntual) como es el caso del Perú con un rango meta de inflación entre 1 y 3\% anual similar al de Australia, Canadá, Israel y Nueva Zelanda. Japón, Suecia e Inglaterra tienen una meta puntual de $2 \%$ lo que equivale a la meta promedio de los países que tienen el rango meta de 1 a $3 \%$. Como se observa en la tabla 1. 


\section{Pensamiento Crítico Vol. 18. Nº I}

La Tabla 2 muestra las tasas de inflación de diversos países en el 2011. Se observa que algunos países han tenido tasas muy altas llegando al 50\% como Bielorusia, Etiopia y Venezuela mientras que otros países tienen tasas de inflación menores a la de Perú.

Tabla 1. Países con Metas de inflación en el Mundo

\begin{tabular}{|c|c|c|}
\hline País & Banco Central & Meta de Inflación \\
\hline Albania & Banco de Albania & $3.0 \%$ \\
\hline Armenia & Banco Central de Armenia & $5.5 \%$ \\
\hline Australia & Banco de Reserva de Australia & $1-3 \%$ \\
\hline Botswana & Banco de Botswana & $3 \%-6 \%$ \\
\hline Brasil & Banco Central de Brasil & $4.5 \%+/-2 \%$ \\
\hline Canadá & Banco de Canadá & $1-3 \%$ \\
\hline Chile & Banco Central de Chile & $3 \%+/-1 \%$ \\
\hline China & Banco de China & $4.0 \%$ \\
\hline Colombia & Banco de la Republica de Colombia & $2-4 \%$ \\
\hline Republica Checa & Banco Nacional Checo & $2.0 \%$ \\
\hline Area Euro & Banco Central Europeo & $<2 \%$ \\
\hline Georgia & Banco Nacional de Georgia & $6.0 \%$ \\
\hline Hungría & Banco Nacional Magyar & $3.0 \%$ \\
\hline Islandia & Banco Central de Islandia & $2.50 \%$ \\
\hline Indonesia & Banco Indonesia & $4.5 \%+/-1 \%$ \\
\hline Israel & Banco de Israel & $1-3 \%$ \\
\hline Jamaica & Banco de Jamaica & $6-8 \%$ \\
\hline Japón & Banco de Japón & $2.0 \%$ \\
\hline México & Banco de México & $3 \%+/-1 \%$ \\
\hline Nueva Zelanda & Banco de Reserva de Nueva Zelanda & $1-3 \%$ \\
\hline Nigeria & Banco Central de Nigeria & $10.0 \%$ \\
\hline Noruega & Banco de Noruega & $2.5 \%$ \\
\hline Perú & Banco Central de Reserva del Peru & $1-3 \%$ \\
\hline Filipines & Banco Central de Filipinas & $4 \%+/-1 \%$ \\
\hline Polonia & Banco Central de Polonia & $2.5 \%+/-1 \%$ \\
\hline Rmania & Banco Nacional de Rumania & $2.5 \%+/-1 \%$ \\
\hline Rusia & Banco de Rusia & $5-6 \%$ \\
\hline Serbia & Banco Nacional Serbia & $4.5 \%+/-1.5 \%$ \\
\hline Sudáfrica & Banco de Reserva de Sudáfrica & $3-6 \%$ \\
\hline Korea del Sur & Banco de Korea & $2-4 \%$ \\
\hline
\end{tabular}




\section{Richard Roca Garay}

Suecia

Suiza

Tailandia

Turkia

Uganda

Reino Unido

Uruguay

EE.UU
Banco Sueco

Banco Nacional Suizo

Banco de Tailandia

Banco Central de Turkia

Banco de Uganda

Banco de Inglaterra

Banco Central del Uruguay

Reserva Federal de EE.UU
$2.0 \%$

$0-2 \%$

$3.0 \%$

$5.0 \%$

$7.0 \%$

$2.0 \%$

$4-6 \%$

$2 \%$

Tabla 2. Inflación por Países 2011

\begin{tabular}{lcc}
\hline Posición & País & Tasa de inflación (\%) \\
\hline 1 & Bielorrusia & 52.4 \\
2 & Etiopía & 33.2 \\
3 & Venezuela & 26.1 \\
32 & Bolivia & 9.9 \\
46 & Haití & 8.5 \\
48 & Rusia & 8.4 \\
49 & Paraguay & 8.3 \\
52 & Nicaragua & 8.1 \\
54 & Uruguay & 8.1 \\
68 & Honduras & 6.8 \\
69 & Brasil & 6.6 \\
77 & Panamá & 5.9 \\
82 & China & 5.5 \\
91 & El Salvador & 5.1 \\
103 & Costa Rica & 4.9 \\
107 & Cuba & 4.7 \\
110 & Ecuador & 4.5 \\
112 & Reino Unido & 4.5 \\
121 & Nueva Zelanda & 4.0 \\
123 & Corea del Sur & 4.0 \\
147 & México & 3.4 \\
149 & Colombia & 3.4 \\
150 & Australia & 3.4 \\
152 & Perú & 3.4 \\
156 & Chile & 3.3 \\
161 & Grecia & 3.1 \\
& España & \\
& & 3 \\
& & \\
& &
\end{tabular}




\section{Pensamiento Crítico Vol. 18. $\mathrm{N}^{\circ}$ I}

\begin{tabular}{lcc}
162 & Estados Unidos & 3.1 \\
163 & Suecia & 3.0 \\
166 & Italia & 2.9 \\
167 & Canadá & 2.9 \\
186 & Francia & 2.3 \\
187 & Alemania & 2.3 \\
208 & Taiwán & 1.4 \\
209 & Noruega & 1.3 \\
218 & Suiza & 0.2 \\
219 & Japón & -0.3 \\
\hline
\end{tabular}

Fuente: CIA World Factbook

La Tabla 3 muestra las tasas de inflación de los países con régimen de metas de inflación en la que el Perú ocupa el lugar número 15 de 23 países lo que muestra claramente que no somos el país con la tasa de inflación más baja del mundo en el periodo 2003-2011.

La tasa de inflación promedio de los países considerados en la tabla 3 es 2.9 mientras que la del Perú es 2.8 lo que la ubica en un nivel intermedio de los países con Metas de Inflación.

Tabla 3. Inflación de Países con Metas de Inflación (2002-2011)

\begin{tabular}{lccccccccccc}
\hline \multicolumn{1}{c}{ PAIS } & $\mathbf{2 0 0 3}$ & $\mathbf{2 0 0 4}$ & $\mathbf{2 0 0 5}$ & $\mathbf{2 0 0 6}$ & $\mathbf{2 0 0 7}$ & $\mathbf{2 0 0 8}$ & $\mathbf{2 0 0 9}$ & $\mathbf{2 0 1 0}$ & $\mathbf{2 0 1 1}$ & PROM & LUGAR \\
\hline Japón & -0.3 & -0.1 & -0.3 & 0.3 & 0.1 & 1.4 & -1.4 & -0.7 & -0.3 & -0.1 & 1 \\
Suiza & 0.6 & 0.9 & 1.2 & 1.2 & 0.7 & 2.4 & -0.5 & 0.7 & 0.2 & 0.8 & 2 \\
Suecia & 1.9 & 0.7 & 0.5 & 1.4 & 2.2 & 3.5 & -0.3 & 1.4 & 3.0 & 1.6 & 3 \\
Alemania & 1.1 & 1.6 & 2.0 & 1.7 & 2.3 & 2.7 & 0.3 & 1.1 & 2.3 & 1.7 & 4 \\
Francia & 2.1 & 2.3 & 1.7 & 1.5 & 1.5 & 2.8 & 0.1 & 1.5 & 2.3 & 1.8 & 5 \\
Israel & 0.7 & 0.0 & 1.3 & -0.1 & 0.5 & 4.6 & 3.3 & 2.6 & 3.5 & 1.8 & 6 \\
Canadá & 2.8 & 1.9 & 2.2 & 2.0 & 2.1 & 1.2 & 0.3 & 1.6 & 2.9 & 1.9 & 7 \\
Noruega & 1.0 & 1.6 & 2.3 & 0.8 & 3.8 & 2.1 & 2.4 & 1.3 & 3.1 & 2.0 & 8 \\
Italia & 2.7 & 2.3 & 2.0 & 2.3 & 1.8 & 3.4 & 0.8 & 1.4 & 2.9 & 2.2 & 9 \\
EE.UU. & 2.3 & 2.5 & 3.2 & 2.5 & 2.9 & 3.8 & -0.3 & 1.4 & 3.1 & 2.4 & 10 \\
Rep Checa & 0.1 & 3.2 & 1.9 & 2.7 & 2.9 & 6.3 & 1.0 & 1.5 & 1.9 & 2.4 & 11 \\
Reino & 1.4 & 1.4 & 2.1 & 3.0 & 2.3 & 3.6 & 2.2 & 3.3 & 4.5 & 2.6 & 12 \\
Unido & 3.0 & 3.2 & 3.4 & 3.5 & 2.8 & 4.1 & -0.3 & 1.3 & 3.1 & 2.7 & 13 \\
España & & & & & & & & & & &
\end{tabular}




\section{Richard Roca Garay}

$\begin{array}{lccccccccccc}\text { Polonia } & 0.7 & 3.4 & 2.2 & 1.3 & 2.5 & 4.2 & 3.5 & 2.6 & 4.3 & 2.7 & 14 \\ \text { Perú } & 2.3 & 3.8 & 1.6 & 2.1 & 1.8 & 5.8 & 2.9 & 1.5 & 3.4 & 2.8 & 15 \\ \text { N. Zelanda } & 1.8 & 2.4 & 3.0 & 3.8 & 2.4 & 4.0 & 2.1 & 2.6 & 4.0 & 2.9 & 16 \\ \text { Australia } & 2.8 & 2.3 & 2.7 & 3.8 & 2.3 & 4.4 & 1.8 & 2.9 & 3.4 & 2.9 & 17 \\ \text { Corea } & 3.6 & 3.6 & 2.8 & 2.2 & 2.5 & 4.7 & 2.8 & 3.0 & 4.0 & 3.2 & 18 \\ \text { Chile } & 2.8 & 2.4 & 3.1 & 2.6 & 4.4 & 8.7 & 1.5 & 1.7 & 3.3 & 3.4 & 19 \\ \text { México } & 4.5 & 5.4 & 4.0 & 3.4 & 4.0 & 5.1 & 3.6 & 4.1 & 3.4 & 4.2 & 20 \\ \text { Colombia } & 7.1 & 5.9 & 5.0 & 4.3 & 5.5 & 7.0 & 4.2 & 3.1 & 3.4 & 5.1 & 21 \\ \text { Brasil } & 14.7 & 7.6 & 6.9 & 3.0 & 3.6 & 5.7 & 4.9 & 4.9 & 6.6 & 6.4 & 22 \\ \text { Turquia } & 25.3 & 9.3 & 8.2 & 9.8 & 8.7 & 10.4 & 6.3 & 8.7 & 6.5 & 10.4 & 23\end{array}$

Fuente: FMI.

La figura 5 muestra la evolución de la inflación mundial desde el 2002 hasta el 2012 comparada con la inflación del Perú. Se puede apreciar que la tasa de inflación (línea roja) peruana esta por debajo de la de America latina y la de los países emergentes pero por encima de los países de Economía Avanzada, de Europa y del G7.

Figura 5. Inflación Mundial del 2002 al 2012

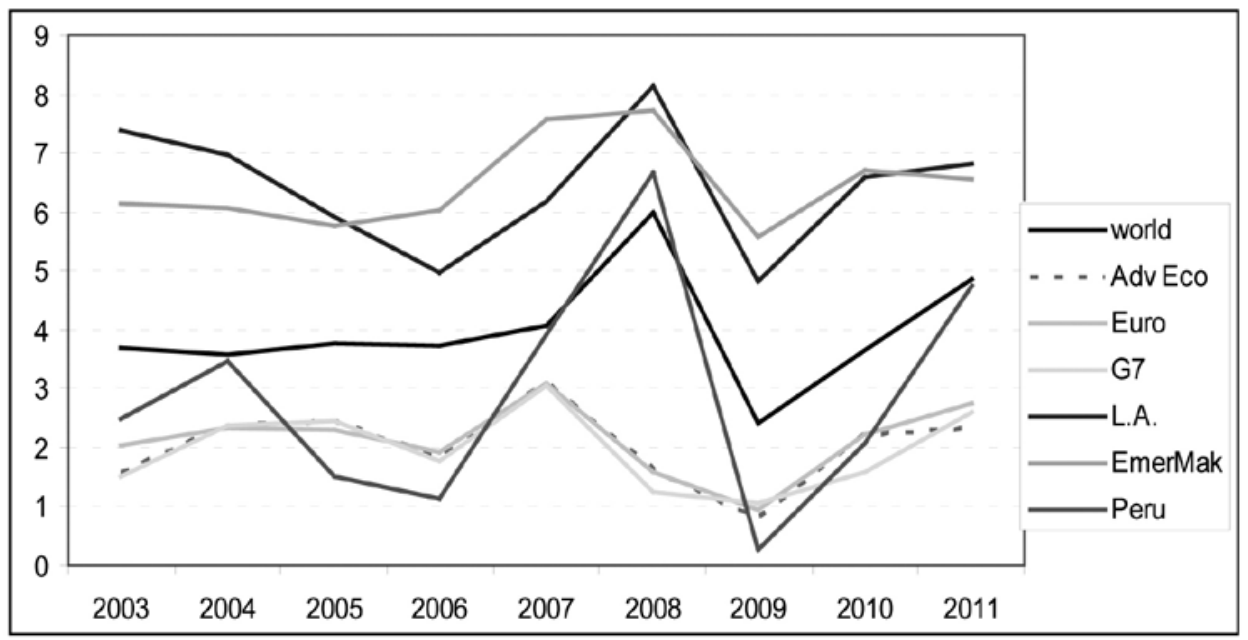

Fuente: FMI World Economic Outlook 2013 abril. 


\section{Pensamiento Crítico Vol. 18. No I}

\section{CONCLUSIONES}

El control de la Inflación es bastante aceptable pues está por debajo del nivel medio de los países con metas de inflación por lo que se puede decir que la efectividad del control de la Inflación en el Perú es aceptable. Ha sido aceptablemente efectiva en controlar la inflación aunque otros países han sido más efectivos pues alcanzaron niveles de inflación más bajos.

\section{REFERENCIAS}

Banco Central de Reserva del Perú (2012) Memorias del BCRP.

Banco Central de Reserva del Perú (2012) Informe de la Inflación. Diciembre.

CIA (2012) World Factbook.

Fondo Monetario Internacional (2013) World Economic Outlook 2013 abril. 